\title{
The risk of asthma is increased among women with polycystic ovary syndrome: a twin study
}

\author{
Louise Zierau ${ }^{1,2,3}$, Howraman Meteran¹, Vibeke Backer ${ }^{1}$, Svend Lindenberg ${ }^{3}$, \\ Axel Skytthe ${ }^{4}$ and Simon Francis Thomsen ${ }^{5,6}$
}

Affiliations: ${ }^{1}$ Respiratory Research Unit, Bispebjerg Hospital, Copenhagen, Denmark. ${ }^{2}$ Faculty of Health and Medical Sciences, University of Copenhagen, Copenhagen, Denmark. ${ }^{3}$ Copenhagen Fertility Center, Copenhagen, Denmark. ${ }^{4}$ The Danish Twin Registry, University of Southern Denmark, Odense, Denmark. ${ }^{5}$ Dept of Dermatology, Bispebjerg Hospital, Copenhagen, Denmark. ${ }^{6}$ Dept of Biomedical Sciences, Faculty of Health and Medical Sciences, University of Copenhagen, Copenhagen, Denmark.

Correspondence: Louise Zierau, Respiratory Research Unit, Dept of Respiratory Medicine L, Bispebjerg University Hospital, Bispebjerg Bakke 23, 2400 Copenhagen NV, Denmark. E-mail: L_Zieraulahotmail.com

\section{ABSTRACT}

Background: Recent registry studies have demonstrated a higher prevalence of asthma among women with polycystic ovary syndrome (PCOS). We aimed to assess the association and heritability of PCOS and asthma in a Danish twin cohort.

Methods: Data for 32382 female twins from the Danish Twin Registry were included. Twins with PCOS were identified by searching the Danish National Patient Registry for International Classification of Diseases-10 code E28.2. Asthma was diagnosed by questionnaires.

Results: 103 (0.3\%) women had a PCOS diagnosis. The risk of asthma was increased among women with PCOS compared with women without (18\% versus 9\%, respectively; OR 2.11 (95\% CI 1.13-3.96); $\mathrm{p}=0.02$ ). After adjustment for age, body mass index, alcohol consumption and smoking status, the risk of asthma was still increased, but was no longer statistically significant (OR 1.54 (95\% CI 0.75-3.17); p=0.24). Variance components analysis showed that shared environmental factors explained $49 \%$ (95\% CI 24-68\%) and unique environmental factors explained 51\% (95\% CI 32-76\%) of the susceptibility to PCOS. For asthma, $44 \%$ (95\% CI $28-61 \%)$ of the variance was explained by genetic factors, whereas $25 \%$ (95\% CI 11-38\%) was ascribable to shared environmental factors and $31 \%$ (95\% CI 26-36\%) to unique environmental factors.

Conclusion: The risk of asthma is twice as high among female twins with PCOS. The individual susceptibility to PCOS is mainly due to environmental factors and not genetics.

@ERSpublications

The association and heritability of polycystic ovary syndrome (PCOS) and asthma was assessed in a Danish twin cohort; the risk of asthma is increased among female twins with PCOS http://bit.ly/2LiyoWR

Cite this article as: Zierau L, Meteran $\mathrm{H}$, Backer V, et al. The risk of asthma is increased among women with polycystic ovary syndrome: a twin study. ERJ Open Res 2019; 5: 00018-2018 [https://doi.org/10.1183/23120541.00018-2018]. 


\section{Introduction}

Polycystic ovary syndrome (PCOS) is the most common endocrine disorder among women of fertile age, affecting as many as $10 \%$ [1]. In addition, asthma is the most common chronic disease in industrialised societies. Women in particular suffer from late-onset asthma and the prevalence of nonatopic asthma is high among adult females [2]. Moreover, both diagnoses can have a massive impact on personal, social and socioeconomic wellbeing, with a negative effect on quality of life $[3,4]$. Despite the high incidence of both asthma and PCOS, little is known about the association and heredity of the two conditions.

The Rotterdam 2003 consensus criteria, used to diagnose PCOS, were first defined almost two decades ago [5]. According to the Rotterdam 2003 consensus, after the exclusion of related disorders, two of the following three criteria must be fulfilled for the diagnosis for PCOS: oligo/anovulation, clinical and/or biochemical hyperandrogenaemia, and presence of polycystic ovaries.

The risk of obesity (metabolic syndrome), cardiovascular disease and diabetes is also increased among women with PCOS [6]. Higher risk of asthma and greater use of asthma and allergy medication have been found among women with PCOS compared with women without PCOS [7, 8]. The pathogenesis of PCOS has not yet been fully elucidated. However, genetics, systemic inflammation [9] and metabolic dysregulation [10] play an important role in PCOS. A study conducted on a Dutch twin population revealed that $66 \%$ of the variation in the susceptibility to PCOS could be explained by genetic factors, whereas $5 \%$ was explained by common environmental factors and $29 \%$ by nonshared environmental factors [11], illustrating the importance of a genetic predisposition in this disorder.

Asthma is characterised by respiratory symptoms along with hyperresponsiveness, inflammation and reversible obstruction of the airways $[12,13]$. As with PCOS, asthma is associated with impaired fertility, probably due to inflammation and metabolic syndrome [14]. Twin studies investigating the heredity of asthma have demonstrated a genetic impact on the risk of asthma development throughout life [15]. Twin studies are a unique method for investigating the contribution of genetic and environmental factors to disease risk in a population.

The aim of this study was to examine the prevalence and association of PCOS and asthma in a large nationwide Danish twin population, and furthermore to estimate the genetic and environmental contributions to PCOS and asthma using the classical twin method.

\section{Methods}

\section{Design}

In this study, data from the Danish Twin Registry and the Danish National Patient Registry were cross-referenced.

\section{The Danish Twin Registry}

The Danish Twin Registry contains data for twins born between 1931 and 2000 in Denmark. Throughout these years three twin cohorts have been included in the registry by completing multidisciplinary questionnaires on lifestyle, health and socioeconomic status. The questionnaires were named OMNIBUS 1994 (twins born 1953-1982; response rate 86\%), OMNIBUS 2002 (twins born 1931-1982; response rate 75\%) and BTU 2003 (twins born 1983-2000; response rate 68\%).

In total, 32382 individual female twins born between 1931 and 2000 were included in our study population (figure 1). Data on asthma diagnosis, smoking and alcohol consumption, body mass index (BMI), and zygosity were included from the OMNIBUS 1994, OMNIBUS 2002 and BTU 2003 questionnaires [16].

\section{Questionnaires}

Asthma

OMNIBUS 1994 identified asthma by the following question: "Have you ever had asthma?" with the response options "yes" or "no". OMNIBUS 2002 identified asthma by the following question: "Do you have, or have you ever had asthma?" with the response options "yes, I have it now", "yes, I have had it" or "no". BTU 2003 identified asthma by the following questions, answered by the parents or by the twin individual, respectively: "Has your child ever had asthma?" and "Have you ever had asthma?" with the response options of leaving a check box marked or unmarked. In all three surveys, asthma was defined by a positive response to the question(s).

The majority of the twins born during 1953-1982 participated in both OMNIBUS 1994 and 2002. If the diagnosis of asthma was present in at least one of the questionnaires, the twin was categorised as having asthma. 


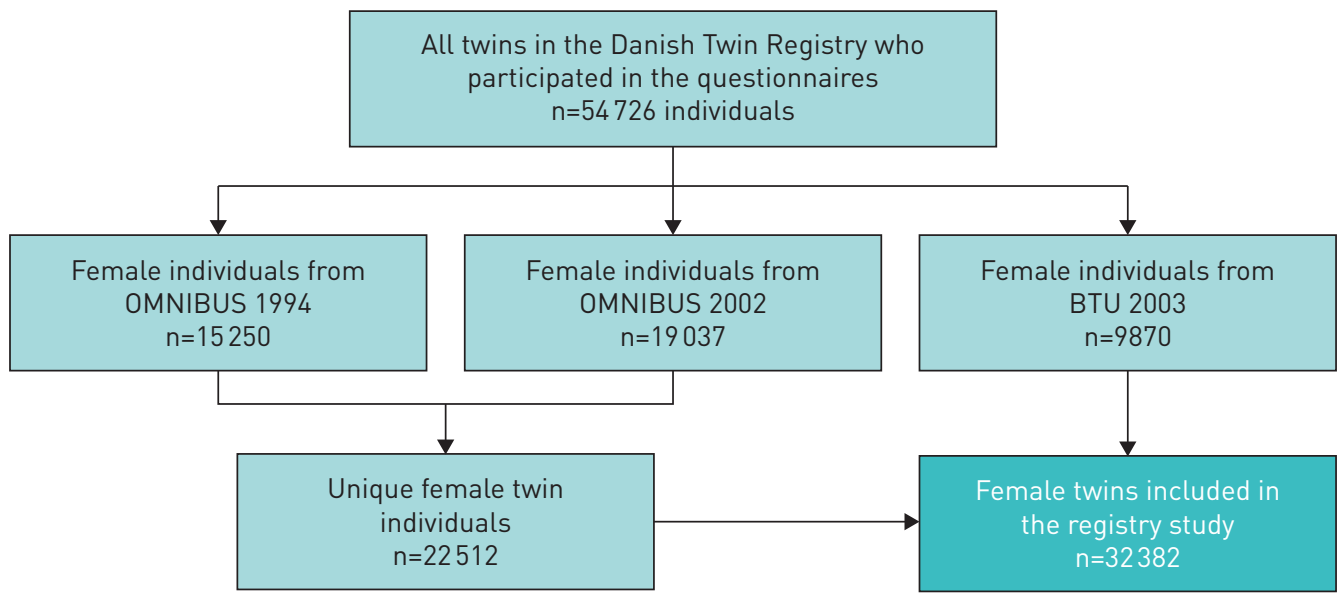

FIGURE 1 Flowchart of study inclusion.

\section{Alcohol consumption}

In OMNIBUS 1994 and 2002, alcohol consumption was based on the following questions: "Do you drink alcohol: yes/no - if "yes", how many units of alcohol (beer, hard liquor, wine and liquor) do you drink each week?”. Alcohol consumption was not addressed in BTU 2003.

\section{Smoking status}

In OMNIBUS 1994 and 2002, smoking status was based on the following categories: never-, ex- or current smoker. Smoking status was not addressed in BTU 2003.

\section{Body mass index}

BMI was calculated as for all subjects $\geqslant 18$ years of age based on self-reported height and weight. For individuals $<18$ years of age the $\mathrm{z}$-score was calculated using the height, weight and age. BMI was divided into four categories: underweight (BMI $<18.5 \mathrm{~kg} \cdot \mathrm{m}^{-2}$ ), normal weight (BMI $18.5-24.9 \mathrm{~kg} \cdot \mathrm{m}^{-2}$ ), overweight (BMI $25.0-29.9 \mathrm{~kg} \cdot \mathrm{m}^{-2}$ ) and obese $\left(\mathrm{BMI} \geqslant 30.0 \mathrm{~kg} \cdot \mathrm{m}^{-2}\right.$ ).

\section{Zygosity}

The zygosity of the twins was determined by four questions of similarity and mistaken identity, which assign zygosity correctly in $>96 \%$ of cases compared with genetic marker information [17].

\section{The twin method}

The principle of the classical twin method is that monozygotic (MZ) twins share all of their genetic makeup, whereas dizygotic (DZ) twins only share half their genes [18]. If MZ twins are more alike for a disease compared with DZ twins, genetic factors can be assumed to contribute to the disease risk. Furthermore, the variation of the phenotypic trait can be divided into an environmental component (shared (C) or unique (E)) and a genetic (A) component according to genetic theory: the ACE model. This model estimates the proportion of the variance explained by genetic factors and the proportion explained by environmental (shared or unique) factors [19].

\section{The Danish National Patient Registry}

The Danish National Patient Registry contains all diagnosed diseases at public hospitals in Denmark since 1977. The register covers both inpatient and outpatient contacts. Between 1977 and 1993 the database used International Classification of Diseases (ICD)-8 codes; from 1994 onwards the database used ICD-10 codes. From 1977 only inpatient contacts were registered, whereas from 1995 the Danish National Patient Registry recorded both inpatient and outpatient contacts [20].

\section{PCOS diagnosis}

The ICD-10 code E28.2 (PCOS) at hospital discharge was used to identify women with PCOS in the Danish National Patient Registry. We used PCOS diagnosis at discharge to ensure that the diagnosis was confirmed, as a diagnosis at the point of admission is often a suspected or working diagnosis. All data were extracted in October 2014. 
Ethics

The Regional Scientific Ethics Committee approved the questionnaire study and the cross-linking of data was approved by the Danish Data Protection Agency (approval 2012-58-0004).

\section{Statistical analysis}

Chi-squared and t-tests were used to compare baseline characteristics in the PCOS and non-PCOS groups. The risk of asthma in patients with PCOS was estimated using multiple logistic regression with age, smoking, alcohol consumption and BMI as covariates. SPSS version 23 (IBM, Armonk, NY, USA) was used for these analyses.

The probandwise concordance $(\mathrm{CPr})$ expresses the probability of one twin being affected given the co-twin is affected. $C \mathrm{Pr}$ is calculated as $2 \times$ number of concordant pairs/ $2 \times$ number of concordant pairs + number of discordant pairs). The proportion of the variation in PCOS susceptibility explained by genetic and environmental factors was estimated from the number of unaffected twins, discordant and concordant pairs included. These estimations were computed using the statistical software $\mathrm{Mx}$ according to the methods described by NEALE and CARDON [21].

\section{Results}

Of the 32382 female twins included in the study, 103 (0.3\%) had the ICD-10 code E28.2 for PCOS. The PCOS group was significantly younger than the non-PCOS group (mean \pm SD $22 \pm 8.2$ versus $31 \pm 18.9$ years; $\mathrm{p}<0.001$ ) and a significantly larger proportion were never-smokers (63\% versus $45 \% ; \mathrm{p}=0.02$ ). The proportion of obese individuals in the PCOS group was also significantly higher $(16 \%$ versus $6 \% ; \mathrm{p}<0.001)$. Alcohol consumption was similar in the two groups (table 1).

The prevalence of asthma was significantly higher in the PCOS group (18\% versus 9\%; OR 2.11 (95\% CI 1.13-3.96); $\mathrm{p}=0.02$ ). After adjustment for age, BMI, alcohol consumption and smoking status, the risk of asthma was still increased, but was no longer statistically significant (OR 1.54 (95\% CI 0.75-3.17); p=0.24) (table 1).

Two of the MZ twin pairs and one DZ twin pair were concordant for PCOS, whereas 33 of the MZ twin pairs and 30 of the DZ twin pairs were discordant for PCOS. The CPr of PCOS was 0.108 among MZ twins and 0.063 among DZ twins ( $p=0.628$ for comparison between MZ and DZ twins). The CPr of asthma was significantly higher among MZ than among DZ twins (0.45 versus $0.30 ; \mathrm{p}<0.001)$ (table 2$)$.

Variance components analysis according to the full ACE model showed that 5\% (95\% CI 0-72\%) of the variance in the susceptibility to PCOS was explained by genetic factors, whereas shared environmental

\section{TABLE 1 Descriptive statistics among female twins with and without polycystic ovary syndrome (PCOS)}

\begin{tabular}{|c|c|c|c|c|c|c|}
\hline & Non-PCOS & PCOS & Crude OR $(95 \% \mathrm{CI})$ & p-value & Adjusted OR $(95 \% \mathrm{CI})^{\#}$ & p-value \\
\hline Subjects & 32279 & 103 & & & & \\
\hline Age $^{\text {ๆ,+ }}$ years & $31 \pm 18.9$ & $22 \pm 8.2$ & $0.92(0.88-0.93)$ & $<0.001$ & $0.99(0.99-0.99)$ & 0.004 \\
\hline$<18.5$ (underweight) & $1269(4)$ & 3 (3) & $0.38(0.05-2.80)$ & 0.35 & $1.13(0.89-1.44)$ & 0.33 \\
\hline $18.5-24.9$ (normal weight) & 21019 (72) & $66(68)$ & Reference & & Reference & \\
\hline $25.0-29.9$ (overweight) & $5151(18)$ & $13(13)$ & $0.98(0.50-1.92)$ & 0.96 & $1.35(1.19-1.54)$ & $<0.001$ \\
\hline Never & $3678(18)$ & $11(18)$ & Reference & & Reference & \\
\hline $1-6$ & 11427 (57) & $39(63)$ & $1.14(0.58-2.23)$ & 0.70 & $0.88(0.77-1.00)$ & 0.06 \\
\hline $7-13$ & $3392(17)$ & $12(19)$ & $1.18(0.52-2.68)$ & 0.69 & $0.89(0.75-1.05)$ & 0.16 \\
\hline $14-20$ & $1101(6)$ & $0(0)$ & 0.00 & 0.99 & $0.92(0.72-1.17)$ & 0.45 \\
\hline$\geqslant 21$ & $393(2)$ & $0(0)$ & 0.00 & 0.99 & $1.00(0.69-1.44)$ & 0.99 \\
\hline \multicolumn{7}{|l|}{ Smoking status ( $n=21839$ ) } \\
\hline
\end{tabular}


TABLE 2 Occurrence and resemblance between female twins for polycystic ovary syndrome (PCOS) and asthma

\begin{tabular}{cccccc} 
Zygosity & Pairs & Discordant pairs & Concordant pairs & CPr & p-value \\
PCOS & & & & & 0.628 \\
MZ & 3625 & 33 & 2 & 0.108 & \\
DZ & 5196 & 30 & 1 & 0.063 & \\
Total & 8821 & 63 & 3 & & $<0.001$ \\
Asthma & & & 147 & 0.45 & \\
MZ & 3570 & 358 & 140 & 0.30 & \\
DZ & 5096 & 664 & 287 & & \\
Total & 8666 & 1022 & & & \\
\hline
\end{tabular}

Data are presented as $\mathrm{n}$, unless otherwise stated. CPr: probandwise concordance; MZ: monozygotic; DZ: dizygotic. $p<0.05$ considered significant.

factors and unique environmental factors explained $45 \%$ (95\% CI 0-68\%) and 50\% (95\% CI 27-76\%) of the variance in susceptibility, respectively. The most parsimonious model was the CE model ( $\mathrm{p}=0.923$ for difference between the ACE and the CE model), in which shared environmental factors explained $49 \%$ (95\% CI 24-68\%) and unique environmental factors explained 51\% (95\% CI 32-76\%) of the variance in the susceptibility to PCOS.

According to the best-fitting model for asthma, 44\% (95\% CI 28-61\%) of the variance in the susceptibility to asthma was explained by genetic factors, whereas $25 \%$ (95\% CI 11-38\%) was ascribable to shared environmental factors and $31 \%$ (95\% CI 26-36\%) to unique environmental factors.

\section{Discussion}

We demonstrated an increased risk of having asthma and PCOS simultaneously. After adjustment for confounders such as age, BMI, smoking and alcohol consumption, the association was statistically insignificant, but still demonstrating an increased OR of 1.54; possibly an association is present but our study lacks power. Furthermore, twin variance components analysis indicated that the individual susceptibility to PCOS was best explained by environmental factors alone. In addition, we confirmed previous findings of a strong genetic component in the susceptibility to asthma.

Interestingly, another large Danish register-based study (of non-twin women) also demonstrated a significantly higher prevalence of asthma among women with PCOS compared with women without PCOS (3\% versus 2.2\%) [7]. This finding was supported by two large Australian register-based studies. In a study by HART and DOCHERTY [22], a significantly higher prevalence of asthma among women with PCOS was found (10.6\% versus 4.5\%; hazard ratio 2.51 ) compared with women without PCOS. These findings show an even higher prevalence of asthma and PCOS in Australian women than in the Danish cohort. A further study using the same cohort demonstrated that pregnant women with PCOS have a significantly higher prevalence of asthma compared with pregnant women without PCOS (13.6\% versus 9.9\%) [23]. Pregnancy in general leads to a higher prevalence of asthma symptoms and asthma exacerbations with emergency visits [24]. One could argue that women with PCOS have an even higher risk of asthma when being pregnant compared with pregnant women without PCOS based on the Australian study.

The possible association of PCOS and asthma could be explained by several factors. First, smoking is known to increase the risk of asthma and the risk of asthma exacerbations [25]. Interestingly, we found a higher prevalence of never-smokers among women with PCOS compared with women without PCOS. As a higher prevalence of asthma is seen among women with PCOS in spite of less smoking, PCOS seems to be a risk factor for asthma independently of smoking status.

The metabolic syndrome and obesity play an important role for both PCOS and asthma, affecting as many as half of patients with PCOS [26] and $11 \%$ of patients with asthma [27]. Weight loss improves symptoms for both groups of patients [28-31]. Thus, being overweight or gaining weight could lead to, or at least affect, the risk of developing asthma [32]. This was reflected in the higher prevalence of obesity and asthma seen among the women with PCOS in our study.

In a recent Australian study, the association of PCOS and asthma remained significant after adjustment for BMI and smoking status, and participants suffering from both diseases were more obese than women with PCOS alone [8]. This illustrates how the metabolic syndrome might increase the risk for the development of asthma among women with PCOS. As the environmental contribution to the development of PCOS, as 
shown in this study, is central, weight control is essential in the prevention of PCOS. However, prospective studies are needed to elucidate the order of events and determine causality.

The possible association of PCOS and asthma could also be due to a common systemic inflammatory pathway. Systemic inflammation in PCOS is characterised by raised levels of C-reactive protein, interleukin-6, tumour necrosis factor, neutrophils and lymphocytes [33, 34]. However, a more specific inflammatory pathway in PCOS has yet to be defined. Conversely, the pathway of systemic inflammation in asthma has been thoroughly investigated, especially the T-helper type 2 (Th2) pathway [35, 36]. Less eosinophilia is seen among asthmatic obese women, indicating a predominantly non-Th2 type of asthma [37]. The idea of a pathophysiological link between PCOS and asthma through the degree of systemic inflammation and metabolic factors is plausible. Systemic inflammation is known to increase with being overweight [38].

Our twin analysis indicated that genetic factors did not contribute to the risk of PCOS, but rather that common environmental factors and unique environmental factors explained the variation in disease risk. These findings are in contrast to the findings of a Dutch twin study [11]. The difference in variance components could be explained by the different diagnostic criteria used in the Dutch study and in our study. In the Dutch study, less than nine menstruations per year and hirsutism or acne were used to define the PCOS diagnosis, whereas we used the ICD code for PCOS, ensuring the most exact patient selection. Given that the environmental contribution to PCOS may have the greatest impact on the disease, this must be incorporated in future research, patient information and disease control to ensure the best possible treatment.

A limitation of our twin study is the low prevalence of PCOS, at $0.3 \%$, which is lower than the $6-10 \%$ observed in the general population [1]. The underestimation of PCOS may be due to a conservative approach and the use of hospital registry information or the fact that many women with PCOS remain undiagnosed for years [39, 40]. Lastly, recall bias regarding asthma, height and weight is a possible limitation of our study as the twin registry is based on self-reported questionnaires. Nevertheless, $89 \%$ of the twins diagnosed through the questionnaires with asthma were subsequently diagnosed with asthma at a clinical examination compared with only $12 \%$ of those with no asthma diagnosis according to the questionnaires [41].

In conclusion, the risk of asthma is twice as high among female twins with PCOS compared with those without PCOS. This study was unable to support the findings of earlier studies that there is a significant genetic susceptibility to PCOS. For asthma, in contrast, genetic factors were a major contributor to the risk of disease. Future clinical and registry studies of the association of PCOS and asthma are needed.

Support statement: This work was supported by a grant from the L.F. Foghts Fond. Funding information for this article has been deposited with the Crossref Funder Registry.

Conflict of interest: L. Zierau reports grants from the L.F. Foghts Fond during the conduct of the study. H. Meteran has nothing to disclose. V. Backer has nothing to disclose. S. Lindenberg has nothing to disclose. A. Skytthe has nothing to disclose. S.F. Thomsen has nothing to disclose.

\section{References}

1 Fauser BCJM, Tarlatzis BC, Rebar RW, et al. Consensus on women's health aspects of polycystic ovary syndrome (PCOS): the Amsterdam ESHRE/ASRM-Sponsored 3rd PCOS Consensus Workshop Group. Fertil Steril 2012; 97: $28-38$.

2 Kwon HL, Belanger K, Bracken MB. Asthma prevalence among pregnant and childbearing-aged women in the United States: estimates from national health surveys. Ann Epidemiol 2003; 13: 317-324.

3 Chen $\mathrm{H}$, Gould MK, Blanc PD, et al. Asthma control, severity, and quality of life: quantifying the effect of uncontrolled disease. J Allergy Clin Immunol 2007; 120: 396-402.

4 Veltman-Verhulst SM, Boivin J, Eijkemans MJC, et al. Emotional distress is a common risk in women with polycystic ovary syndrome: a systematic review and meta-analysis of 28 studies. Hum Reprod Update 2012; 18: 638-651.

5 Rotterdam ESHRE/ASRM-Sponsored PCOS Consensus Workshop Group. Revised 2003 consensus on diagnostic criteria and long-term health risks related to polycystic ovary syndrome (PCOS). Hum Reprod 2004; 19: 41-47.

6 Scicchitano P, Dentamaro I, Carbonara R, et al. Cardiovascular risk in women with PCOS. Int J Endocrinol Metab 2012; 10: 611-618.

7 Glintborg D, Rubin KH, Nybo M, et al. Morbidity and medicine prescriptions in a nationwide Danish population of patients diagnosed with polycystic ovary syndrome. Eur J Endocrinol 2015; 172: 627-638.

8 Htet TD, Teede HJ, de Courten B, et al. Asthma in reproductive-aged women with polycystic ovary syndrome and association with obesity. Eur Respir J 2017; 49: 1601334.

9 Tarkun I, Cetinarslan B, Türemen E, et al. Association between circulating tumor necrosis factor-alpha, interleukin-6, and insulin resistance in normal-weight women with polycystic ovary syndrome. Metab Syndr Relat Disord 2006; 4: 122-128.

10 Daan NMP, Louwers YV, Koster MPH, et al. Cardiovascular and metabolic profiles amongst different polycystic ovary syndrome phenotypes: who is really at risk? Fertil Steril 2014; 102: 1444-1451. 
11 Vink JM, Sadrzadeh S, Lambalk CB, et al. Heritability of polycystic ovary syndrome in a Dutch twin-family study J Clin Endocrinol Metab 2006; 91: 2100-2104.

12 Holgate ST. Innate and adaptive immune responses in asthma. Nat Med 2012; 18: 673-683.

13 Lai CKW, Beasley R, Crane J, et al. Global variation in the prevalence and severity of asthma symptoms: phase three of the International Study of Asthma and Allergies in Childhood (ISAAC). Thorax 2009; 64: 476-483.

14 Gade EJ, Thomsen SF, Lindenberg S, et al. Asthma affects time to pregnancy and fertility: a register-based twin study. Eur Respir J 2014; 43: 1077-1085.

15 Thomsen SF, van der Sluis S, Kyvik KO, et al. Estimates of asthma heritability in a large twin sample. Clin Exp Allergy 2010; 40: 1054-1061.

16 Skytthe A, Kyvik KO, Holm NV, et al. The Danish Twin Registry. Scand J Public Health 2011; 39: 7 Suppl., 75-78.

17 Christiansen L, Frederiksen H, Schousboe K, et al. Age- and sex-differences in the validity of questionnaire-based zygosity in twins. Twin Res 2003; 6: 275-278.

18 Hawkes CH. Twin studies in medicine: what do they tell us? Q J Med 1997; 90: 311-321.

19 Posthuma D, Beem AL, de Geus EJC, et al. Theory and practice in quantitative genetics. Twin Res 2003; 6: 361-376.

20 Lynge E, Sandegaard JL, Rebolj M. The Danish National Patient Register. Scand J Public Health 2011; 39: 30-33.

21 Neal MC, Cardon LR. Methodology for Studies of Twins and Families. NATO ASI Series. Dordrecht, Kluwer, 1990.

22 Hart R, Doherty DA. The potential implications of a PCOS diagnosis on a woman's long-term health using data linkage. J Clin Endocrinol Metab 2015; 100: 911-919.

23 Doherty DA, Newnham JP, Bower C, et al. Implications of polycystic ovary syndrome for pregnancy and for the health of offspring. Obstet Gynecol 2015; 125: 1397-1406.

24 Kwon HL, Triche EW, Belanger K, et al. The epidemiology of asthma during pregnancy: prevalence, diagnosis, and symptoms. Immunol Allergy Clin North Am 2006; 26: 29-62.

25 Jayes L, Haslam PL, Gratziou CG, et al. SmokeHaz: systematic reviews and meta-analyses of the effects of smoking on respiratory health. Chest 2016; 150: 164-179.

26 Velez LM, Motta AB. Association between polycystic ovary syndrome and metabolic syndrome. Curr Med Chem 2014; 21: 3999-4012.

27 Akinbami LJ, Fryar CD. Current Asthma Prevalence by Weight Status Among Adults: United States, 2001-2014. NCHS Data Brief 239. Hyattsville, National Center for Health Statistics, 2016.

28 Pasquali R, Gambineri A, Biscotti D, et al. Effect of long-term treatment with metformin added to hypocaloric diet on body composition, fat distribution, and androgen and insulin levels in abdominally obese women with and without the polycystic ovary syndrome. J Clin Endocrinol Metab 2000; 85: 2767-2774.

29 Barber TM, McCarthy MI, Wass JA, et al. Obesity and polycystic ovary syndrome. UK Clin Endocrinol 2006; 65: 137-145.

30 Panidis D, Tziomalos K, Papadakis E, et al. Lifestyle intervention and anti-obesity therapies in the polycystic ovary syndrome: impact on metabolism and fertility. Endocrine 2013; 44: 583-590.

31 Hakala K, Stenius-Aarniala B, Sovijärvi A. Effects of weight loss on peak flow variability, airways obstruction, and lung volumes in obese patients with asthma. Chest 2000; 118: 1315-1321.

32 Bildstrup L, Backer V, Thomsen SF. Increased body mass index predicts severity of asthma symptoms but not objective asthma traits in a large sample of asthmatics. J Asthma 2015; 52: 687-692.

33 Duleba AJ, Dokras A. Is PCOS an inflammatory process? Fertil Steril 2012; 97: 7-12.

34 Küçük M, Altınkaya SÖ, Nergiz S, et al. Interleukin-6 levels in relation with hormonal and metabolic profile in patients with polycystic ovary syndrome. Gynecol Endocrinol 2014; 30: 423-427.

35 Kudo M, Ishigatsubo Y, Aoki I. Pathology of asthma. Front Microbiol 2013; 4: 263.

36 Rothenberg ME. Eosinophilia. N Engl J Med 1998; 338: 1592-1600.

37 Haldar P, Pavord ID, Shaw DE, et al. Cluster analysis and clinical asthma phenotypes. Am J Respir Crit Care Med 2008; 178: 218-224.

38 Ellulu MS, Khaza'ai H, Rahmat A, et al. Obesity can predict and promote systemic inflammation in healthy adults. Int J Cardiol 2016; 215: 318-324.

39 Christensen SB, Black MH, Smith N, et al. Prevalence of polycystic ovary syndrome in adolescents. Fertil Steril 2013; 100: 470-477.

40 Shannon M, Wang Y. Polycystic ovary syndrome: a common but often unrecognized condition. J Midwifery Womens Health 2012; 57: 221-230.

41 Thomsen SF, van der Sluis S, Kyvik KO, et al. A study of asthma severity in adult twins. Clin Respir J 2012; 6: 228-237. 